\title{
BMJ Open Analysis of the comprehensive non- pharmaceutical interventions and measures in containing the COVID-19 epidemic in Shenzhen: a retrospective study
}

\author{
Shule Xu, ${ }^{1}$ Peiyi Liu, ${ }^{1}$ Shujiang Mei, ${ }^{1}$ Qiuying Lv, ${ }^{1}$ Cong Cheng, ${ }^{1}$ Yan Lu, ${ }^{1}$ \\ Dongfeng Kong, ${ }^{1}$ Xiaoliang Wu, ${ }^{1}$ Ying Wen, ${ }^{1}$ Bin Cao, ${ }^{1}$ Shitong Gao, ${ }^{1}$ \\ Huawei Xiong, ${ }^{1}$ Jin Zhao, ${ }^{2}$ Yuanyuan Huang, ${ }^{3}$ Yijuan Luo, ${ }^{2}$ Tiejian Feng ${ }^{1}$
}

To cite: Xu S, Liu P, Mei S, et al. Analysis of the comprehensive non-pharmaceutical interventions and measures in containing the COVID-19 epidemic in Shenzhen: a retrospective study. BMJ Open 2021:11:e044940. doi:10.1136/ bmjopen-2020-044940

- Prepublication history for this paper is available online. To view these files, please visit the journal online (http://dx.doi. org/10.1136/bmjopen-2020044940).

SX and PL contributed equally.

Received 25 September 2020 Accepted 29 June 2021

Check for updates

(C) Author(s) (or their employer(s)) 2021. Re-use permitted under CC BY-NC. No commercial re-use. See rights and permissions. Published by BMJ.

${ }^{1}$ Department of Communicable Disease Control and Prevention, Shenzhen Center for Disease Control and Prevention, Shenzhen, Guangdong, China ${ }^{2}$ Department of AIDS Control and Prevention, Shenzhen Center for Disease Control and Prevention, Shenzhen, Guangdong, China ${ }^{3}$ Department of School Health, Shenzhen Center for Disease Control and Prevention, Shenzhen, Guangdong, China

Correspondence to

Dr Tiejian Feng;

fengtiej@126.com

\section{ABSTRACT}

Objective To analyse the epidemiological characteristics of family clusters of COVID-19 and the three stages of the comprehensive non-pharmaceutical interventions and measures implemented in Shenzhen.

Methods The epidemic curve of COVID-19 was drawn and the impact of the comprehensive non-pharmaceutical interventions and measures was analysed by the different periods of the epidemic.

Results A total of 427 cases ( 417 confirmed cases and 10 asymptomatic infectious cases) were reported in Shenzhen, of which 259 (60.7\%) were clustered cases. 97 cluster events were drawn and most cluster events $(97.3 \%)$ occurred in families. There were three stages of the COVID-19 epidemic in Shenzhen. The epidemic increased rapidly, but the peak lasted for a short time, while the decline in incidence was rapid and large. Conclusions Family clusters were the main feature of the COVID-19 outbreak in Shenzhen in 2020, and the Shenzhen government rolled out a quick response to the epidemic. Non-pharmaceutical interventions and measures were proven to have effectively contained community transmission, limit the transmission to aggregation and reduce the scale of transmission within a household.

\section{INTRODUCTION}

Since the outbreak of COVID-19 in Wuhan by the end of 2019, it has spread fast to other areas of China and overseas within several months in 2020. ${ }^{1}$ COVID-19 has been identified by the WHO as a public health emergency of international concern. ${ }^{2}$ According to the epidemiological characteristics of COVID-19 during the different time periods, all provinces in China have adopted targeted preventive strategies. ${ }^{3}$ Shenzhen city, a special economic zone in southern Guangdong Province, has the third largest container port in the world. The city has a population of over 13 million, of which about $65 \%$ were non-registered
Strengths and limitations of this study

- We have drawn the epidemic curve of COVID-19 divided into three stages and analysed its trend.

- This study summarised the main interventions and measures in different populations.

- The observed cases were incomplete due to the ongoing epidemic, it might not show the whole characteristics of the cluster events very well.

- The study sample size is small and there are geographical limitations.

migrant population (the so-called floating population). The population density is as large as 6500 people per square kilometre. In this environment, Shenzhen city was at a relatively high risk of importation of COVID19, with the first imported case in Guangdong Province reported in Shenzhen. At the end of February, the number of confirmed cases of COVID-19 in Shenzhen reached 427, most of which were from Hubei Province.

The epidemic in Wuhan aroused the professional vigilance of people in Shenzhen city, and the operation of the current 'flu surveillance system' and 'pneumonia of unexplained aetiology surveillance system' was strengthened before the epidemic in Shenzhen. On 8 January 2020, the first case of pneumonia of unexplained aetiology was found in Shenzhen city. After diagnostic detection and confirmation by the Guangdong Center for Disease Control and Prevention (CDC) and China CDC, the first case of COVID-19 was officially confirmed on 19 January 2020, which rang alarm bells in Shenzhen city. This was the first case of a personto-person transmission as a family cluster, published and cited by scholars and shared 
with the academic community in China and overseas. ${ }^{1}$ However, comprehensive non-pharmaceutical interventions and measures were regularly released in Shenzhen city, including full incorporation and immediate detection of suspected cases, rapid diagnosis and case isolation, and rigorous tracking and quarantine of close contacts. The rapid and appropriate public health emergency response for the first case in Shenzhen city not only initiated the prevention and control of COVID-19, but also provided solid scientific evidence for the government to perform risk assessment and implement strategies.

The epidemiological characteristics of COVID-19 have been widely reported, ${ }^{4-9}$ while the evaluation and analysis of non-pharmaceutical interventions and measures, as an important part of epidemiology, have rarely been reported. In this study, we conducted a detailed review of the epidemiological investigation report of each case in Shenzhen city and analysed the impact of the nonpharmaceutical interventions and measures on the transmission process by different stages of the epidemic.

\section{METHODS}

\section{Study design and data sources}

We conducted a retrospective study of confirmed cases of COVID-19 that emerged in Shenzhen city from 14 January to 29 February 2020 which were reported in China Information System for Disease Control and Prevention. We also review in detail the field epidemiological investigation report.

\section{Definition of clusters}

Common exposure to COVID-19 in this study referred to cases who were exposed to the environment with SARS-CoV-2 or have a history of direct contact with the same confirmed case outside Shenzhen city. Local close contacts referred to those who were exposed to risk factors in Shenzhen city. The simultaneous occurrence of more than two cases within a family or class or unit was defined as a cluster event. Family cluster events were events of transmission occurring in a household and involved family members, relatives or housekeepers. Events that occurred in a class or unit such as a travel group, meeting, dining party and so on were considered non-family cluster events. Moreover, mixed cluster events with cases including family members and non-family members (relatives, friends or even colleagues) were also mentioned in our study.

\section{Classification of three stages}

Based on the key interventions and measures against the COVID-19 epidemic, and combined with the time distribution of COVID-19 cases in Shenzhen city, the outbreak of COVID-19 was classified into three stages by the important dates that could affect virus transmission: (1) the time before 23 January 2020, when level 1 emergency response (dual control of Wuhan city and Guangdong Province) was not activated; (2) the period of 23-30
January 2020, when the peak of the COVID-19 outbreak in Shenzhen city was recorded: on 28 January 2020, the headquarters of the epidemic prevention and control in Shenzhen city's CDC were set up, centralised quarantine points were started to quarantine close contacts, and furthermore COVID-19 screening of patients started in fever clinics; and (3) considering the lagging effect of policy, the third stage of outbreak was the day after 30 January 2020.

\section{Statistical analysis}

Descriptive analysis was used to describe the demographic characteristics, along with the distribution of clustered cases. Change in trend of the COVID-19 epidemic curve in Shenzhen from 25 December 2019 to 28 February 2020 is displayed in a histogram. Cases were diagnosed in accordance with the guidelines of the national prevention and control, and all cases included in the analysis were confirmed cases. According to the results of epidemiological investigation, cases were divided into clustered cases and non-clustered cases. All statistical analyses were performed using Microsoft Excel V.2017.

\section{Patient and public involvement \\ No patients were involved.}

\section{RESULTS}

Characteristics of clustered cases with COVID-19 in Shenzhen city

Among 417 COVID-19 confirmed and 10 asymptomatic infectious cases, $259(60.7 \%)$ were clustered cases, of which 252 cases were family clusters (figure 1A). Up to 29 February 2020, there were 97 cluster events of COVID-19, of which 59 events included 2 people (118 people), 20 events included 3 people (60 people), 9 events included 4 people (36 people), 4 events included 5 people (20 people), and 3 events included 6 people (18 people) (figure 1B). Among the family clustered cases, the proportion of imported cases with coexposure was $65.2 \%$ (figure 1C). Only one case was a non-family cluster and three were within mixed cluster events involving seven non-family members, of which four cases were imported from Wuhan. Those were in clusters 25, 34, 64 and 48, who had contact with friends, colleagues and other travellers, respectively (figure 2).

\section{Epidemic trends in cluster outbreak of COVID-19 in Shenzhen city}

The epidemic curve of the cluster outbreak of COVID-19 is presented in figure 3 . Based on the important dates that could affect virus transmission and cluster outbreak, we classified the period into three stages. Since the first case in Shenzhen city was found on 1 January 2020, the period before 23 January 2020 was the stage of increasing cases of COVID-19 and there were about two long incubation periods (14 days). Cases peaked and plateaued between 24 and 30 January, and had been steadily declining since 
A

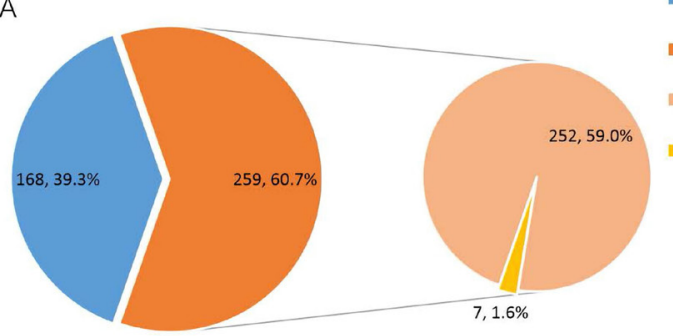

B

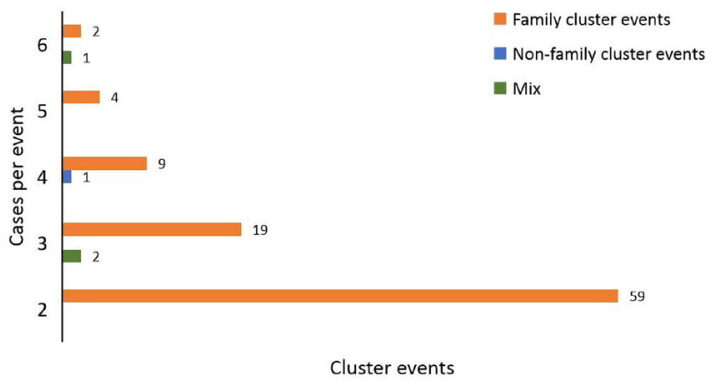
cases

Non-cluster cases

Cluster cases

Family cluster cases

Non-family cluster

C

Co-exposure

- Local close contacts

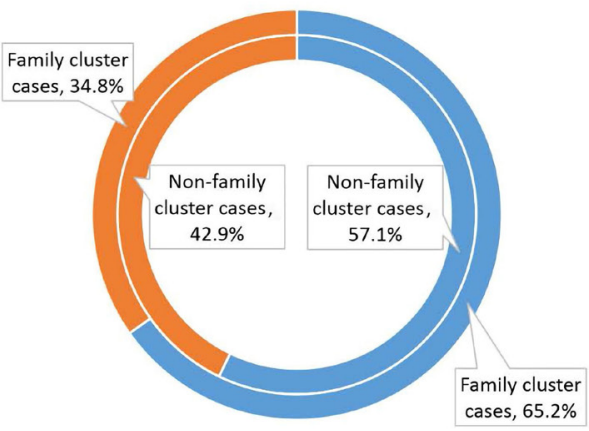

Figure 1 Distribution of clustered cases with COVID-19 in Shenzhen City. (A) Chart of clustered cases constituent ratio among a total of 427 cases in Shenzhen City. (B) Distribution and composition of clustered epidemics. (C) Proportion of imported cases in family clustered cases and non-family clustered cases.

then. The third stage showed a declining trend from 30 January to 14 February, which was a long incubation period of COVID-19.

\section{Comprehensive non-pharmaceutical interventions and measures}

To make the different interventions and measures more effective and efficient, we roughly divided the population of Shenzhen into four categories and took the different interventions and measures by group (table 1 ).

\section{DISCUSSION}

In the past several years, respiratory infectious diseases such as the different subtypes of avian influenza A viruses, severe acute respiratory syndrome coronavirus and Middle East respiratory syndrome coronavirus have emerged continuously and have become a public health concern. ${ }^{10-14}$ Since the outbreak of COVID-19 by the end of 2019, the pandemic has caused enormous health, economic and societal impacts globally.

Based on the epidemiological characteristics observed on all the confirmed cases and close contacts so far in Shenzhen city, most locally infected cases were clustered and especially occurred within households. Although cluster events could have occurred through multiple ways of contact, such as contact within households, companies, schools, dorms, etc, or contact during travelling, family cluster events were the main feature of COVID-19 outbreak in Shenzhen city (figure 1). Thus, limiting transmission within households and reducing its scale in order to contain community transmission is the most important target of prevention and control.

As shown in figure 3, the epidemic of COVID-19 in Shenzhen experienced rising, plateauing and declining stages.
The number of cases increased rapidly at the beginning, with a short duration of peak period. In general, the prevalence of COVID-19 in Shenzhen was characterised by a rapid increase at the beginning of the epidemic, while the peak duration was short and followed a steady decline, to end within a short period. Although the epidemic curve showed the phenomenon of human-to-human transmission, 'trailing' was not obvious, suggesting that the interventions and measures were effective. A study in Shenzhen adopted the expected number of COVID-19 cases obtained from the SEIAR (Susceptible, Exposed, Infectious, Asymptomatic, Recovered) model, compared the epidemic process with the actual situation, and found that the actual total number of cases decreased by $69.8 \%$. The decrease in local cases was even greater, which suggests that the interventions and measures had obvious effects. ${ }^{15}$

During the first stage, since the outbreak of pneumonia with unknown aetiology in the late December 2019 in Wuhan, the government began tracking its spread and started to prepare the necessary responses. The Shenzhen CDC activated the emergency response measures of disease control system on 10 January 2020. Subsequently, the first case was diagnosed on 14 January 2020. The joint prevention and control mechanism for major infectious diseases was initiated. Meanwhile the dual control plan in Wuhan city was activated, and the level 1 (highest level) response with comprehensive interventions and measures was activated in Shenzhen city. As for the individual level, because COVID-19 appears to be transmitted via direct contact with respiratory droplets and fomites during close and unprotected contact, temperature monitoring, hand washing, wearing of mask, health declarations and monthlong 'stay at home' measures were fundamentally applied. 


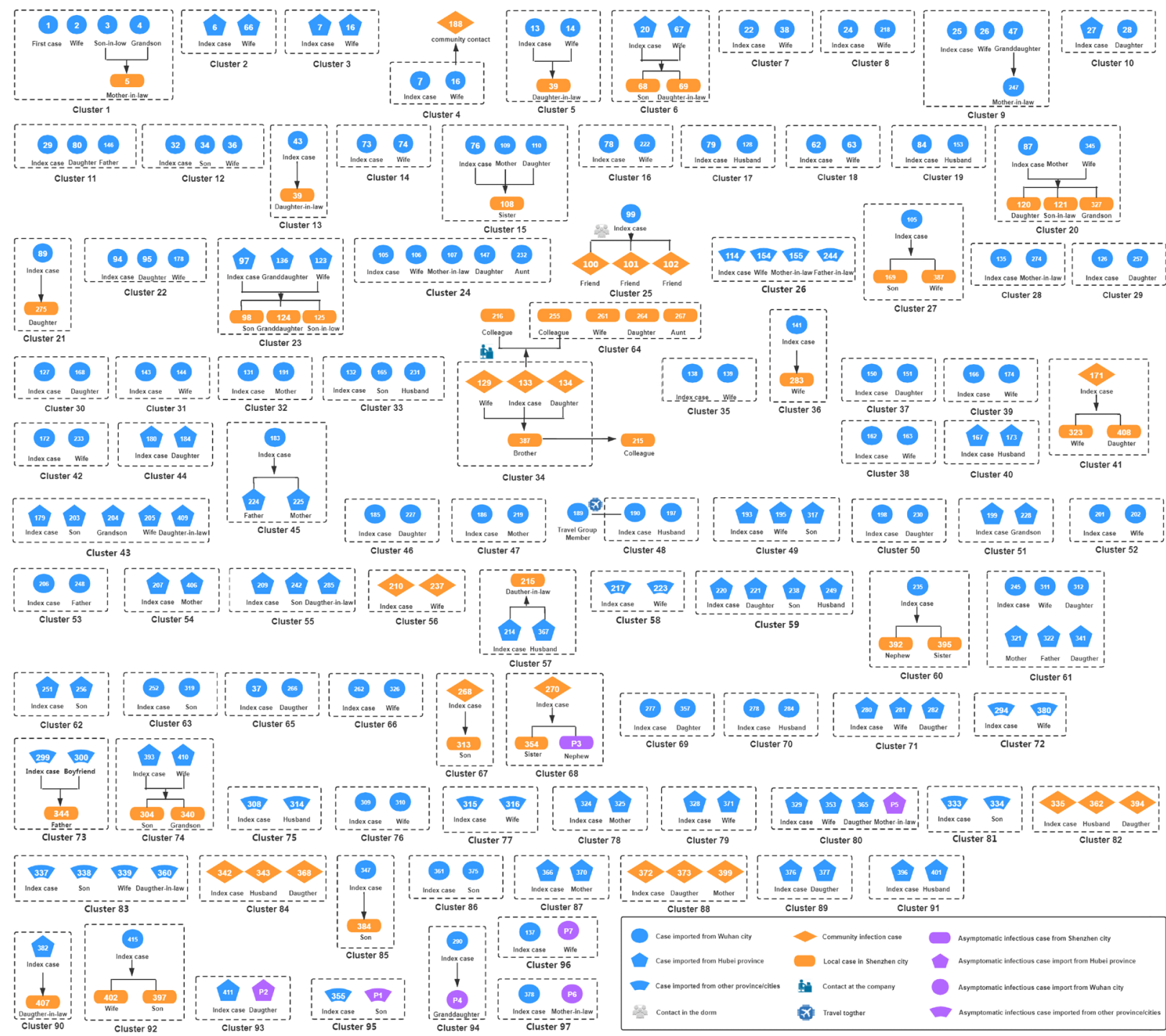

Figure 2 Cluster events of COVID-19 in Shenzhen City, 29 February 2020 ( $n=97)$. Coexposures are depicted in blue, local cases are depicted in orange, and asymptomatic infectious cases are depicted in purple.

As for the public, the main interventions were suspension and cancellation of public and mass gathering activities, travel prohibitions, and control of transportation and traffic. As for health professionals, prevention and control mainly centred on rapid diagnosis, isolation of suspected cases, surveillance of contacts, contact tracing and quarantine measures. In order to strengthen public risk communications and health education, detailed information on prevention and control measures and the epidemic was regularly released on the website of Shenzhen Municipal Health Commission. On 28 January 2020, the headquarters of the epidemic prevention and control in Shenzhen CDC were set up. Moreover, 11 centralised quarantine points started to quarantine close contacts in 10 districts of the city, which was proven to be an effective and special intervention to reduce and control transmission. In addition, COVID-19 screening of patients was carried out in fever clinics on 30 January 2020. Therefore, the optimal strategy is to take strong measures to target the source of infection, to provide preventive disinfection in key sites and to improve the capacity of people to protect themselves. The WHO refers to these as non-pharmacological interventions, also called containment strategy. ${ }^{3}$

This study summarises the main interventions and measures taken by 4 groups. Compared with the prevalence and the controlled COVID-19 situation in China, Guangdong Province and other provinces, ${ }^{3}$ Shenzhen strengthened its professional disease surveillance system at the early stage of the epidemic and enhanced the awareness of community health service centres and CDC professionals in all districts, which facilitated the early detection of the first case of COVID-19. Moreover, case investigation in designated hospitals and medical units initiated by the health administration department, especially the joint prevention and control mechanism, had laid the foundation for the government-led emergency response. After the level 1 response, community prevention and control were in place, and high-tech forces such as risk identification, personnel migration and contact tracing are precise prevention and control measures 


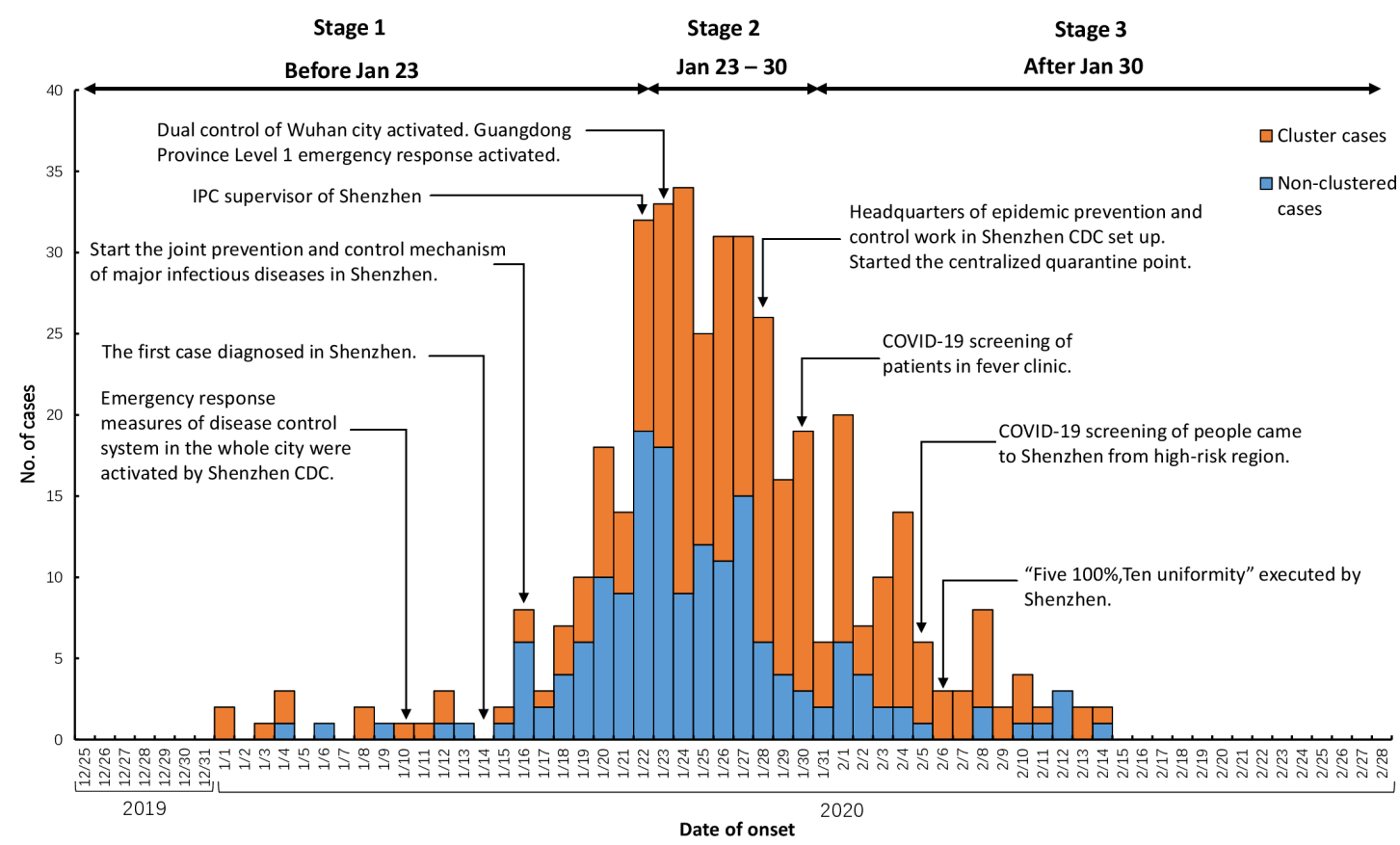

Figure 3 Daily cases of COVID-19 onset and the important control measures across different periods in Shenzhen city. CDC, Center for Disease Control and Prevention; IPC, Infection Prevention and Control.

that are proven effective. A study showed that if level 1 response was not initiated on 23 January, more than three times as imported and local cases were expected to occur as actual cases by 7 March $2020 .{ }^{15}$ In addition, legal disclosure of case information and places of stay promoted by the health authority had increased the transparency and pertinence of prevention and control measures to the public. With the global spread of COVID-19, epidemiological characteristics have become clearer, and intervention strategies such as early detection of cases and isolation for treatment and significantly reducing the chance of human-to-human transmission have been emphasised. ${ }^{16}$ Interventions and measures are also suggested to lower COVID-19 morbidity and mortality, as well as prevent large-scale spread of cases. ${ }^{17}$

In this study, epidemiological theories and methods were adopted to explore the prevention and control strategies and measures for COVID-19 in Shenzhen. However,

Table 1 Main prevention and control measures of COVID-19 for different populations in Shenzhen

\begin{tabular}{|c|c|c|}
\hline Classification & Main prevention and control measures & Common measures \\
\hline $\begin{array}{l}\text { COVID-19 } \\
\text { cases }\end{array}$ & $\begin{array}{l}\text { Increased the capacity of designated hospitals for } \\
\text { medical treatment. } \\
\text { Built backup hospital for medical treatment. } \\
\text { Legal disclosure of information on place of stay and } \\
\text { epidemiological investigation of cases. }\end{array}$ & $\begin{array}{l}\text { Nucleic acid screening for outpatients with fever, } \\
\text { centralised quarantined observers and home quarantine } \\
\text { medical observers were carried out comprehensively. } \\
\text { Level } 1 \text { emergency response (dual control of Wuhan city } \\
\text { and Guangdong Province) was activated. }\end{array}$ \\
\hline Close contacts & $\begin{array}{l}\text { Established centralised isolation points for close } \\
\text { contacts. }\end{array}$ & $\begin{array}{l}\text { Implemented the policy of free of charge, including } \\
\text { treatment of patients, medical observation of close }\end{array}$ \\
\hline General public & $\begin{array}{l}\text { A combination of community workstations, } \\
\text { community health services and community police to } \\
\text { contain community infection and spread. } \\
\text { Restriction on social distancing, suspension of } \\
\text { travel agencies and cruise ships, and closure of } \\
\text { sightseeing sites. } \\
\text { Close management of the whole community. } \\
\text { Extended the Spring Festival holiday, postponed } \\
\text { the start of the school year, and resumed work and } \\
\text { factories in an orderly manner. }\end{array}$ & \\
\hline
\end{tabular}


there are several limitations. First, the study sample size is limited and is only representative of a similar geographical area. Second, the study only roughly divides the epidemic curve into three phases; phased model may be needed to make quantitative evaluation of the effect of continuously strengthening prevention and control measures.

Contributors Conceptualisation: SX, PL, TF, SM. Formal analysis: SX, PL. Funding acquisition: TF. Methodology: SX, PL, QL, CC. Analysis and interpretation of data: YanL, DK, XW. Resources: YW, BC, SG, HX, JZ, YH, YijL. Supervision: TF, SM. Validation: SX, PL, TF, SM. Writing-original draft: SX, PL. Writing-review and editing: TF, SM.

Funding This study was supported by the Key Project of Shenzhen Science and Technology Innovation Commission (no. 202002073000003 for TF).

Competing interests None declared.

Patient consent for publication Not required.

Ethics approval This study was approved by the Shenzhen Center for Disease Control and Prevention Ethics Committee (no. QS2020050025).

Provenance and peer review Not commissioned; externally peer reviewed. Data availability statement № data are available.

Open access This is an open access article distributed in accordance with the Creative Commons Attribution Non Commercial (CC BY-NC 4.0) license, which permits others to distribute, remix, adapt, build upon this work non-commercially, and license their derivative works on different terms, provided the original work is properly cited, appropriate credit is given, any changes made indicated, and the use is non-commercial. See: http://creativecommons.org/licenses/by-nc/4.0/.

\section{REFERENCES}

1 Organization WH. Novel coronavirus (2019-nCoV) situation reports, 2020. Available: https://www.who.int/docs/default-source/ coronaviruse/situation-reports

2 World Health Organization. A Joint Statement on Tourism and COVID-19-UNWTO and WHO Call for Responsibility and Coordination [EB/OL], 2020. Available: https://www.who.int/newsroom/detail/27-02-2020-a-joint-statement-on-tourism-and-covid-19unwto-and-who-call-for-responsibility-and-coordination
3 World Health Organization. Report of the WHO-China joint mission on coronavirus disease 2019 (Covid-19) 2020 [EB/OL], 2020. Available: https://www.who.int/publications-detail/report-of-the-whochina-joint-mission-on-coronavirus-disease-2019-(covid-19)

$4 \mathrm{Hu}$ B, Guo H, Zhou P, et al. Characteristics of SARS-CoV-2 and COVID-19. Nat Rev Microbiol 2021;19:141-54.

5 Dong Y, Mo X, Hu Y, et al. Epidemiology of COVID-19 among children in China. Pediatrics 2020;145:e20200702.

6 Jiang F, Deng L, Zhang L, et al. Review of the clinical characteristics of coronavirus disease 2019 (COVID-19). J Gen Intern Med 2020;35:1545-9.

7 Tu H, Tu S, Gao S, et al. Current epidemiological and clinical features of COVID-19; a global perspective from China. $J$ Infect 2020;81:1-9.

8 Chan JF-W, Yuan S, Kok K-H, et al. A familial cluster of pneumonia associated with the 2019 novel coronavirus indicating personto-person transmission: a study of a family cluster. Lancet 2020;395:514-23.

9 Ghinai I, McPherson TD, Hunter JC, et al. First known person-to-person transmission of severe acute respiratory syndrome coronavirus 2 (SARS-CoV-2) in the USA. Lancet 2020;395:1137-44.

10 Paules $\mathrm{Cl}$, Marston HD, Fauci AS. Coronavirus Infections-More than just the common cold. JAMA 2020;323:707.

11 Chowell G, Bertozzi SM, Colchero MA, et al. Severe respiratory disease concurrent with the circulation of H1N1 influenza. N Engl $J$ Med 2009;361:674-9.

12 Fraser C, Donnelly CA, Cauchemez S, Hanage WP, et al. Pandemic potential of a strain of influenza A (H1N1): early findings. Science 2009;324:1557-61.

13 Guan Y, Peiris JSM, Zheng B, Poon LL, et al. Molecular epidemiology of the novel coronavirus that causes severe acute respiratory syndrome. Lancet 2004;363:99-104.

$14 \mathrm{Kim} \mathrm{KH}$, Tandi TE, Choi JW, Moon JM, et al. Middle East respiratory syndrome coronavirus (MERS-CoV) outbreak in South Korea, 2015: epidemiology, characteristics and public health implications. $J$ Hosp Infect 2017;95:207-13.

15 Zou X, Wu YS, Liu XJ, Xuan Z, Yongsheng W, Xiaojian L, et al. [Evaluation of the emergency response strategies and measures on the epidemic of COVID-19 in Shenzhen, China]. Zhonghua Liu Xing Bing Xue Za Zhi 2020;41:1225-30.

16 Rothan HA, Byrareddy SN. The epidemiology and pathogenesis of coronavirus disease (COVID-19) outbreak. J Autoimmun 2020;109:102433.

17 Wilder-Smith A, Chiew CJ, Lee VJ. Can we contain the COVID-19 outbreak with the same measures as for SARS? Lancet Infect Dis 2020;20:e102-7. 